\title{
Two progressed malignant phyllodes tumors of the breast harbor alterations in genes frequently involved in other advanced cancers
}

\author{
Mattea Reinisch ${ }^{1 *} \mathbb{D}$, Sherko Kuemmel ${ }^{1,2} \mathbb{D}$, Elisabeth Breit ${ }^{1} \mathbb{D}$, Ingo Theuerkauf ${ }^{3}$, Hakima Harrach ${ }^{1}$, \\ Dorothea Schindowski ${ }^{1}$, Detlef Moka ${ }^{4}$, Marcus Bettstetter ${ }^{5}$, Simona Bruzas ${ }^{1}$ and Ouafaa Chiari ${ }^{1}$
}

\begin{abstract}
Background: The genomic landscape of phyllodes tumors (PTs) of the breast is not well defined, especially in patients with advanced disease. To shed light on this topic, paired primary and progressed tumor samples from two patients with malignant PTs were subjected to next-generation sequencing (NGS) followed by functional analysis of genetic alterations using two prediction tools.

Methods: The DNA of both the primary tumor and distant metastases of Patient 1 and the primary and recurrent tumor of Patient 2 were subjected to molecular profiling. NGS with the FoundationOne ${ }^{\circledR}$ assay was performed in a commercial molecular pathology laboratory. Two in silico prediction tools were used to estimate the pathogenicity of indicated genetic alterations.

Results: In total, 38 genomic alterations were detected, of which 11 were predicted to be probably benign. In Patient 1,14 aberrations were identified in the primary tumor and 17 in pulmonary metastases, 12 of which were identical. In the primary and recurrent tumor of Patient 2,17 and 15 sequence variants, respectively, were found, with 13 overlapping findings. Affected genes included seven (TP53, TERT, APC, ARID1A, EGFR, KMT2D, and RB1) of the top 10 most frequently altered genes in other advanced cancer entities, as well as four actionable therapeutic targets (EGFR, KIT, PDGFRA, and BRIP1). Of note, seven genes coding for receptor tyrosine kinases were affected: three in Patient 1 and four in Patient 2. Several genes (e.g. EPHA3, EPHA7, and EPHB1) were shown to be altered for the first time in PTs.
\end{abstract}

Conclusions: The two progressed malignant PTs investigated here share some of the major genetic events occurring in other advanced cancers.

Keywords: Malignant phyllodes tumor, Breast, Next-generation sequencing, Advanced cancer, Metastasis, Molecular profiling

\section{Background}

Phyllodes tumors (PTs) of the breast are rare fibroepithelial neoplasms composed of connective tissue stroma and epithelial elements [1, 2]. The mean age at diagnosis is around 40 years [1]. PTs represent up

\footnotetext{
*Correspondence: M.Reinisch@kem-med.com

1 Breast Unit, Kliniken Essen-Mitte, 45136 Essen, Germany

Full list of author information is available at the end of the article
}

to $0.5 \%$ of all breast tumors and are categorized into benign, borderline, and malignant subtypes. Malignant PTs (MPTs) account for $10-15 \%$ of all PT cases [3], and patients with MTPs have a 5-year survival rate of about $54 \%$ [4]. Some patients present with a rapidly growing MTP [5]. Recurrent disease occurs in 23-30\% [1] and distant metastases in 9-27\%, mainly in MPTs [6]. Patients with metastatic MPTs have a very poor prognosis and may not respond well to standard systemic original author(s) and the source, provide a link to the Creative Commons licence, and indicate if changes were made. The images or other third party material in this article are included in the article's Creative Commons licence, unless indicated otherwise in a credit line to the material. If material is not included in the article's Creative Commons licence and your intended use is not permitted by statutory regulation or exceeds the permitted use, you will need to obtain permission directly from the copyright holder. To view a copy of this licence, visit http://creativecommons.org/licenses/by/4.0/. The Creative Commons Public Domain Dedication waiver (http://creativeco mmons.org/publicdomain/zero/1.0/) applies to the data made available in this article, unless otherwise stated in a credit line to the data. 
therapy; the duration of survival after diagnosis of distant disease ranged from 1 to 41 months [6]. The mainstay of treatment for MPTs is local excision of the tumor, aiming to achieve wide negative margins. Systemic adjuvant therapy is not generally recommended for PTs, mainly due to a lack of supporting clinical studies [7]; however, metastatic PTs are treated like soft tissue sarcomas $[1,8]$ and should therefore receive one or more lines of chemotherapy.

The pathogenesis and underlying genomic landscape of PTs are poorly understood, especially for metastatic disease $[9,10]$. The application of commercially available, advanced DNA sequencing technologies has enabled standardized investigation of the mutational status of several hundred cancer-related genes in PTs during the last 8 years and has provided new information on this topic [9-18]. In addition to genetic alterations in classic tumor suppressor genes (TSGs; e.g. TP53, $R B 1$ ) or oncogenes (e.g. EGFR), hotspot mutations in PIK3CA [12], the TERT promoter region [18, 19], and MED12 [9, 14] have frequently been reported. Genetic alterations including loss-of-function alterations in TP53 and RB1 might have a potential driver function in MPTs [11]. It has also been suggested that TERT promoter mutations, either alone [14] or in combination with MED12 mutations [15], play an important role in the etiology and progression of PTs. Moreover, several less-characterized genetic alterations (e.g. in ATRX [16, 17], BCORL1 [9], and ZNF217 [11]) whose role in the development and progression of PTs is unknown were identified. As alterations in those genes were rarely reported they are probably not one of the key drivers in the development of PTs. Aside from purely researchdriven motivations, a refinement of the genomic profile and subsequent identification of drug targets could create an opportunity for personalized therapy [8].

However, a major challenge in applying next-generation sequencing (NGS) for clinical diagnostics and therapeutic decision-making is the interpretation of identified genetic alterations, in particular variants of unknown significance (VUS) [20, 21]. Several web-based databases (e.g. Catalogue of Somatic Mutations in Cancer, COSMIC [22]), computational algorithms for predicting the impact of mutations on amino acid sequence and protein function (e.g. MutationTaster2 [23]) and knowledge bases incorporating clinical and experimental evidence (e.g. ClinVar [24]) are available free of charge and could aid in the analysis of genetic variants.

Here we present molecular profiling of two patients with MPTs, one with distant and the other with locally recurrent disease. NGS, followed by functional analysis $[23,25]$ of indicated aberrations, was performed for primary and matched progressed tumor specimens.

\section{Materials and methods}

\section{Patients}

Patient 1, a 55-year-old postmenopausal woman from Kazakhstan, presented herself to our institution one month after she had undergone mastectomy of the right breast and axillary lymph node dissection (Fig. 1) in Kazakhstan where the tumor was initially diagnosed as a triple-negative breast sarcoma (pT2m, pN0 [0/3], cM0) without lymphovascular invasion. She was in good general condition (Karnofsky Performance Status: 100\%) and had no family history of PT, although her mother had died of pancreatic cancer at the age of 78. A histological review of slides from the primary tumor (Ki67 index of 70\%) indicated a MPT with pleomorphic stromal cells, showing brisk mitotic activity and invasive margins. In the submitted tissue blocks, there was no epithelial or heterologous (e.g. liposarcoma or chondrosarcoma) component. Diagnosis of MPT was made after exclusion of other spindle cell lesions, especially spindle cell carcinoma. Positron emission tomography (PET)/ computed tomography (CT) revealed several pulmonary nodules, located in the right upper and lower lobe, which were subsequently excised by a video-assisted wedge resection. Postoperative classification was pM1 (PUL) and was followed by palliative chemotherapy with epirubicin and cyclophosphamide. Three months later, a second PET/CT indicated progressive pulmonary metastatic disease in the right and left lobe. Thereafter, treatment was changed to paclitaxel with bevacizumab. Four months later, the patient presented with complete clinical remission of metastatic disease; however, after a further 3 months, CT of the chest again indicated progressive disease (two pulmonary nodules in the right upper lobe, one nodule in the right lower lobe, and one nodule in the left lower lobe), and the patient began gemcitabine and carboplatin treatment. Under this regimen, she had stable disease for 6 months but due to the progression of the metastatic disease, treatment was changed to eribulin. Thereafter, the patient returned to Kazakhstan and was lost to follow-up.

Patient 2, a 29-year-old premenopausal woman, was in good general condition and had no significant medical history, although an aunt had died of metastatic breast cancer at the age of 45 . A cystic mass of $30 \times 25 \mathrm{~mm}$ was detected on mammogram images (Fig. 2). Thereafter, lumpectomy of the right breast with wide margins was performed, followed by reconstructive surgery with defect coverage using medial and caudal rotations flaps. Histological analysis of the surgical specimen indicated a regressive, cystic, partly necrotic MPT, measuring up to $38 \mathrm{~mm}$ in its greatest dimension (pT2). Seven axillary lymph nodes were pathologically negative. There were no distant metastases (cM0), nor was there lymphovascular 


\begin{tabular}{|c|c|c|}
\hline Time & Treatment/action & Diagnosis/results $\longrightarrow$ \\
\hline $11 / 2017$ & Mammography (Kazakhstan) & Not available \\
\hline $12 / 2017$ & $\begin{array}{l}\text { Mastectomy of the right breast } \\
\text { (Kazakhstan) }\end{array}$ & $\begin{array}{l}\text { Bifocal triple-negative, invasive breast sarcoma of the right breast: pT2m (max. } 30 \\
\mathrm{~mm} \text { ), pNO (0/3), cM0 }\end{array}$ \\
\hline $\begin{array}{l}01 / 2018 \\
\quad \prod\end{array}$ & $\begin{array}{l}\text { Second opinion (Germany) } \\
\text { Positron emission tomography } \\
\text { (PET)/computed tomography (CT) }\end{array}$ & $\begin{array}{l}\text { Malignant phyllodes tumor with sarcomatoid differentiation } \\
\text { Pulmonary, polytopic metastases }\end{array}$ \\
\hline $\begin{array}{c}02 / 2018 \\
\prod\end{array}$ & $\begin{array}{l}\text { Wedge resection with removal of } \\
\text { a 4-mm pulmonary nodule from } \\
\text { the right lower lobe and two 5-mm } \\
\text { nodules from the right upper lobe } \\
\text { Histological evaluation }\end{array}$ & $\begin{array}{l}\text { Pleomorphic stromal cells in the lung } \\
\text { metastasis, similar to the primary } \\
\text { tumor, with marked atypia and } \\
\text { increased mitotic activity (hemato- } \\
\text { xylin and eosin, x 200) }\end{array}$ \\
\hline $02 / 2018$ & NGS of lung metastasis & Tumor mutational burden: intermediate; $\varepsilon$ \\
\hline $\begin{array}{l}02-05 / \\
2018\end{array}$ & Palliative chemotherapy & 4x epirubicin/cyclophosphamide $(90 / 600$ \\
\hline $05 / 2018$ & $\mathrm{PET} / \mathrm{CT}$ of the chest & $\begin{array}{l}\text { Progressive metastatic disease with } \\
\text { new pulmonary nodules }\end{array}$ \\
\hline & & $\begin{array}{l}\text { Response Evaluation Criteria in } \\
\text { Solid Tumors (RECIST): } 1.1\end{array}$ \\
\hline $\begin{array}{l}05-12 / \\
2018\end{array}$ & Palliative therapy & 18x paclitaxel $\left(80 \mathrm{mg} / \mathrm{m}^{2}\right) \mathrm{q} 1 \mathrm{w}+9 x$ bevacizumab $(15 \mathrm{mg} / \mathrm{kg})$ \\
\hline $09 / 2018$ & $\mathrm{PET} / \mathrm{CT}$ & $\begin{array}{l}\text { Remission of pulmonary lesions with } \\
\text { substantial decrease in size and } \\
\text { metabolic uptake }\end{array}$ \\
\hline $12 / 2018$ & $\mathrm{PET} / \mathrm{CT}$ & Progressive disease with new multiple hypermetabolic pulmonary nodules \\
\hline $12 / 2018$ & Palliative chemotherapy & $6 x$ gemcitabine $\left(1000 \mathrm{mg} / \mathrm{m}^{2}\right) \mathrm{d} 1+\mathrm{d} 8 \mathrm{q} 3 \mathrm{w}+$ carboplatin AUC $4 \mathrm{q} 3 \mathrm{w}$ \\
\hline $03 / 2019$ & $\mathrm{PET} / \mathrm{CT}$ & Stable disease \\
\hline $06 / 2019$ & $\mathrm{PET} / \mathrm{CT}$ & Progressive pulmonary disease \\
\hline $06 / 2019$ & Palliative chemotherapy & Eribulin $1.23 \mathrm{mg} / \mathrm{m}^{2} \mathrm{~d} 1+8 ; \mathrm{q} 21$ \\
\hline $07 / 2019$ & NGS of untreated primary tumor & Tumor mutational burden: low; 5 mutations/Mb; 14 genetic alterations \\
\hline
\end{tabular}

Fig. 1 Medical history of a 55-year woman with a metastatic malignant phyllodes tumor (MPT). The patient from Kazakhstan was initially diagnosed with an invasive sarcoma, which was later identified as a MPT following review in the Breast Unit of the Kliniken Essen-Mitte (KEM). In 2009, she had undergone adnexectomy due to the presence of an ovarian cyst. During her examination at the KEM, several pulmonary nodules were detected. Excision and histological characterization of the nodules revealed distant metastases of the MPT. The patient received several lines of chemotherapy treatment and the angiogenesis inhibitor bevacizumab. After her last appointment at the KEM in 06/2019, the patient was lost to follow-up. Next-generation sequencing (NGS) of the metastatic and primary tumor samples was performed 


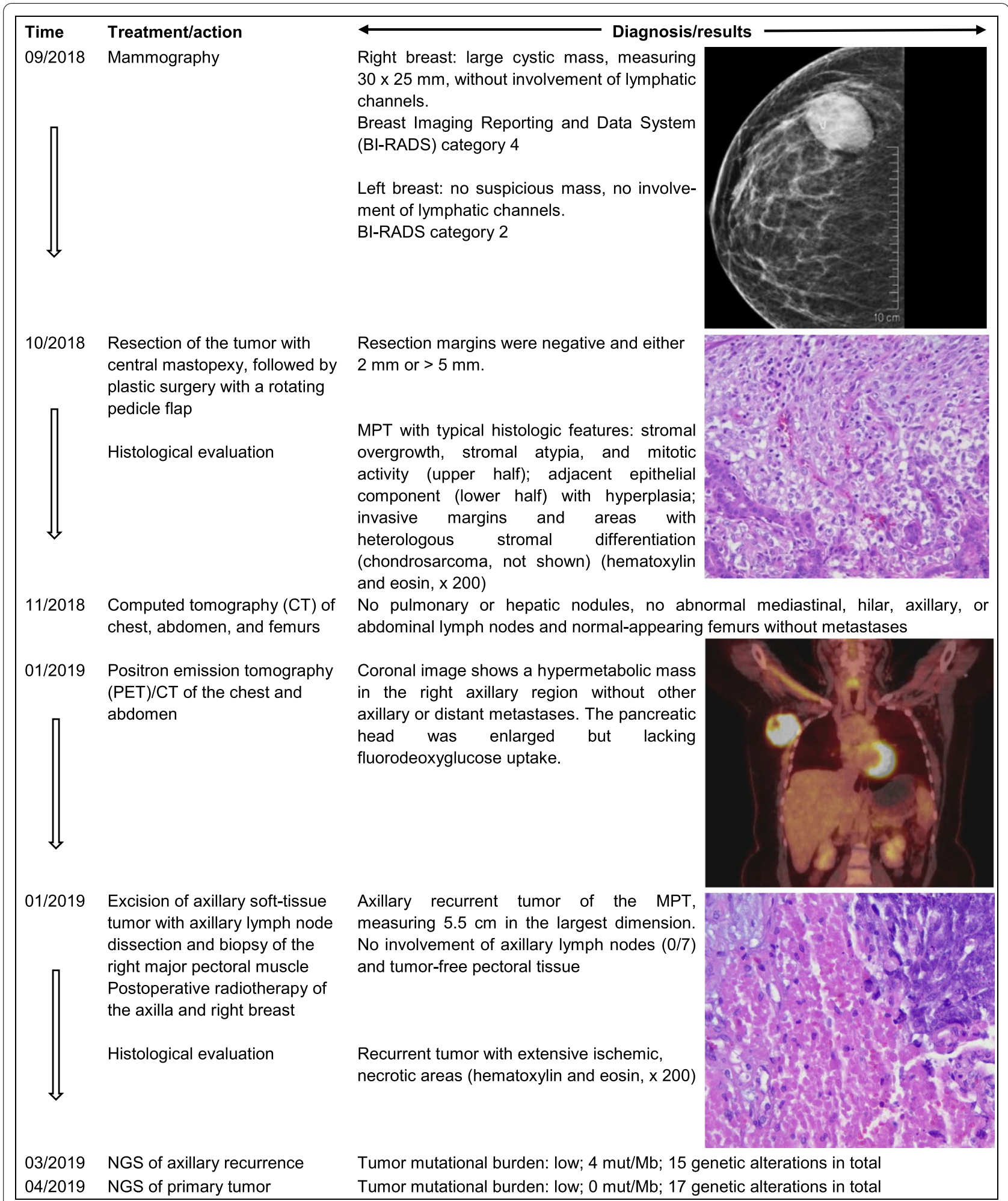

Fig. 2 Medical history of a 29-year woman with a recurrent malignant phyllodes tumor (MPT) of the breast (Patient 2). The young woman presented with a lesion in the right breast on mammogram images. Following excision of the lesion, reconstructive surgery was performed. The mass was identified as a MPT without regional or distant spread. Three months later, PET/CT scans demonstrated a large mass in the axillary region. After surgical excision of the lesion and axillary lymph nodes, a node-negative recurrent tumor of the MPT was diagnosed. Adjuvant radiotherapy of the right breast and axilla was administered. Next-generation sequencing (NGS) was performed for both the primary and recurrent MPT. Follow-up examinations showed no local or distant recurrence to date (April 2021) 
invasion (L0, V0). Due to insufficient safety margins of less than $10 \mathrm{~mm}$, re-excision surgery had to be performed. Repeated CT scans demonstrated that the chest, abdomen, axilla, and femurs were disease-free. Three months after the primary surgery, PET/CT indicated a large, hypermetabolic mass in the axillary region. Subsequent excision and histological characterization of the lesion $(5.5 \mathrm{~cm})$ identified recurrence of the MPT. The patient received postoperative radiotherapy of the right breast and axilla. Regular follow-up exams with ultrasound, mammography, and magnetic resonance imaging did not indicate progression of the disease.

\section{Ethics statement}

According to $\$ 15$ of the Nordrhein-Westfalen (Germany) Medical Association professional code of conduct, retrospective studies do not require ethics committee approval. Patients provided written informed consent.

\section{Genomic profiling}

Formalin-fixed, paraffin-embedded specimens of the primary tumor (P1) and pulmonary metastases (M1) of Patient 1 , and the primary (P2), and recurrent tumor (R2) of Patient 2 were analyzed in a commercial molecular pathology laboratory (Molekularpathologie Südbayern, Penzberg, Germany). Extracted DNA was subjected to NGS utilizing the hybrid capture-based FoundationOne $^{\circledR}$ (sample M1) or FoundationOne ${ }^{\circledR}$ CDx (samples P1, P2, R2) assay (Foundation Medicine Inc., Cambridge, MA, US) as previously described [26]. Focused sequencing with the FoundationOne ${ }^{\circledR}$ assay was conducted for exons of 315 genes and introns of 28 genes, and with the FoundationOne ${ }^{\circledR}$ CDx assay for exons of 324 genes and introns of 36 genes, frequently associated with various neoplasms. The indicated genomic regions were analyzed for base substitutions, insertions and deletions; copy number alterations; rearrangements, translocations; microsatellite instability; and tumor mutational burden (TMB). The routine result report contained a listing of identified gene alterations. Upon request, we received the coding DNA reference sequence, the transcript number, and chromosomal position, as these details were needed for the analysis of sequence variants with in silico prediction tools.

\section{In silico prediction tools}

FATHMM-XF [25] and MutationTaster2 [23] were applied in order to predict the functional effects of identified genetic alterations. FATHMM-XF can be used for functional analysis of non-synonymous single-nucleotide variants (SNVs) and MutationTaster2 can be applied to SNVs as well as insertions and deletions. Predictions with FATHMM-XF are expressed as p-values (range, 0-1) with values close to 0 or 1 yielding predictions with the highest accuracy; values $>0.5$ indicate a deleterious SNV and those $<0.5$ a neutral or benign SNV. MutationTaster2 predicts pathogenicity of genetic variants as one of four possible types as described in the following types: disease-causing, probably deleterious; disease-causing automatic, known to be deleterious; polymorphism, probably harmless; polymorphism automatic, known to be harmless. To evaluate mutations, chromosomal location (FATHMM-XF) or the position of altered bases in the gene and Ensembl transcript ID (MutationTaster2) were investigated. The Molekularpathologie Südbayern provided relevant data that are not part of a regular FoundationOne ${ }^{\circledR}$ or FoundationOne ${ }^{\circledR}$ CDx report.

\section{Results}

\section{NGS of paired tumor samples}

Alterations in oncogenes or TSGs are depicted in Table 1; alteration in genes, which have not yet been clearly identified as TSGs or oncogenes, are listed in Table 2, with additional details provided in Additional file 1: Table S1. VUS were assigned according to the FoundationOne ${ }^{\circledR}$ (CDx) report. The four investigated MPT samples were microsatellite stable. Samples from Patient 1 were compared with each other; similarly, samples from Patient 2 were compared with each other.

The untreated primary tumor (P1) and lung metastases (M1) of Patient 1 had a TMB of 5 mut/Mb and 8 mut/Mb, respectively. Fourteen (P1) and 17 (M1) genetic aberrations were identified, of which 12 were present in both lesions (Tables 1 and 2, Fig. 3). P1 and M1 had identical alterations in several TSGs $(A P C, B R I P 1, K M T 2 D$, and TP53), oncogenes (CDK4 and MED12), and genes with dual roles in activating or suppressing carcinogenesis such as GRIN2A and TERT (Table 1). SNVs predicted by MutationTaster2 to be probably diseasecausing were identified in $A P C, C D K 4, M E D 12, K M T 2 D$, TP53, PLCG2, ZNF703, and receptor tyrosine kinases (RTKs) EPHA3 (P1/M1), EPHB1 (P1/M1), and EPHA7 (M1). Functional Analysis through Hidden Markov Models (FATHMM)-XF predicted four of these to be benign. ARID1A, EPHA7, GRIN2A, PLCG2, and SPTA1 were altered exclusively in M1, and MAF and CASP8 in P1 (Fig. 3). Both the primary tumor and the pulmonary metastasis harbored a missense mutation in the start codon (p.M1V) of BRIP1. Whereas in February 2018 no therapeutic option was indicated, the NGS report from July 2019 recommended off-label treatment with olaparib, which had by then been approved for metastatic breast cancer. At around this time the patient returned to Kazakhstan and was lost to follow-up. It is therefore not clear if she received NGS-based therapy. 


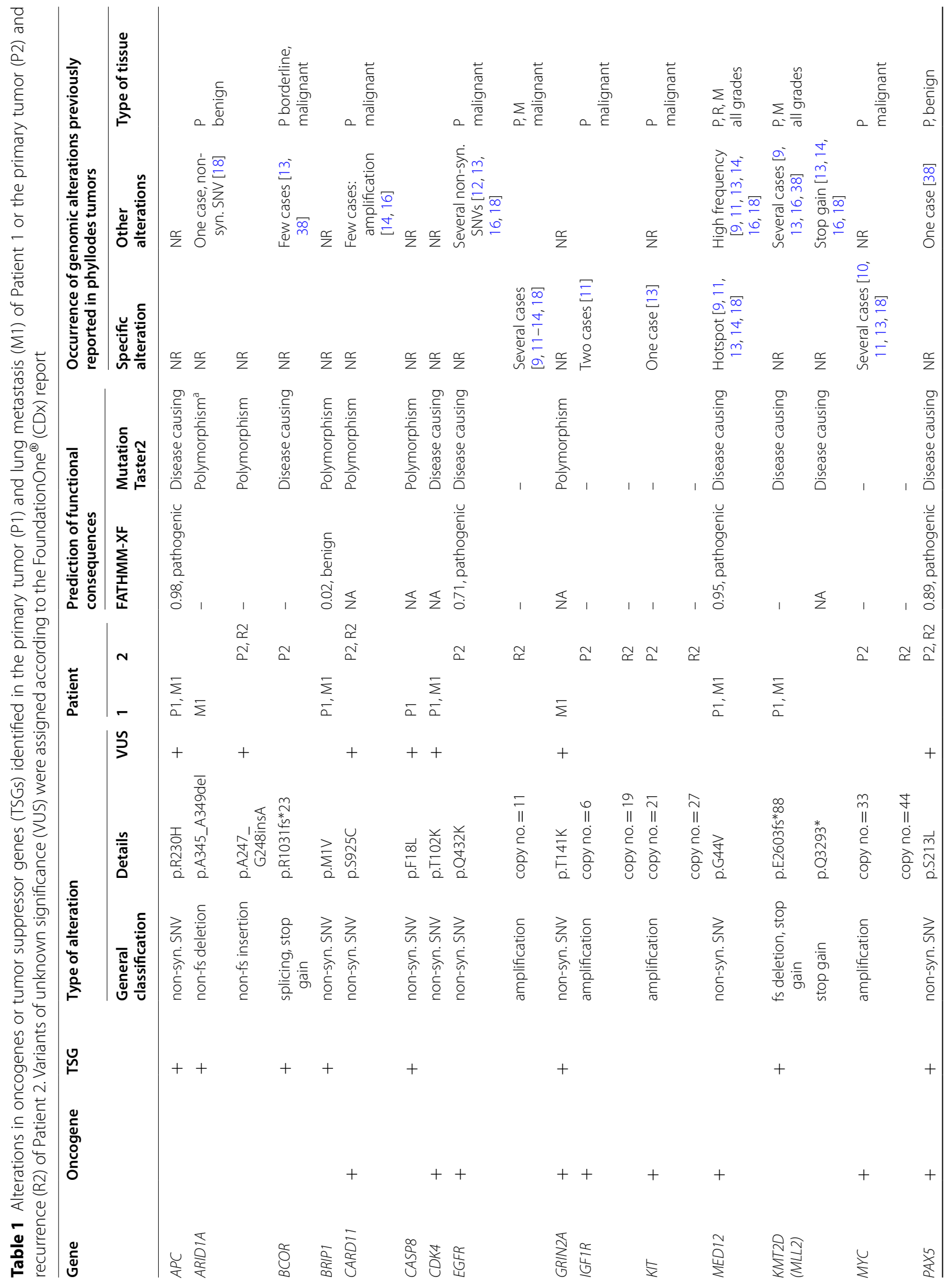




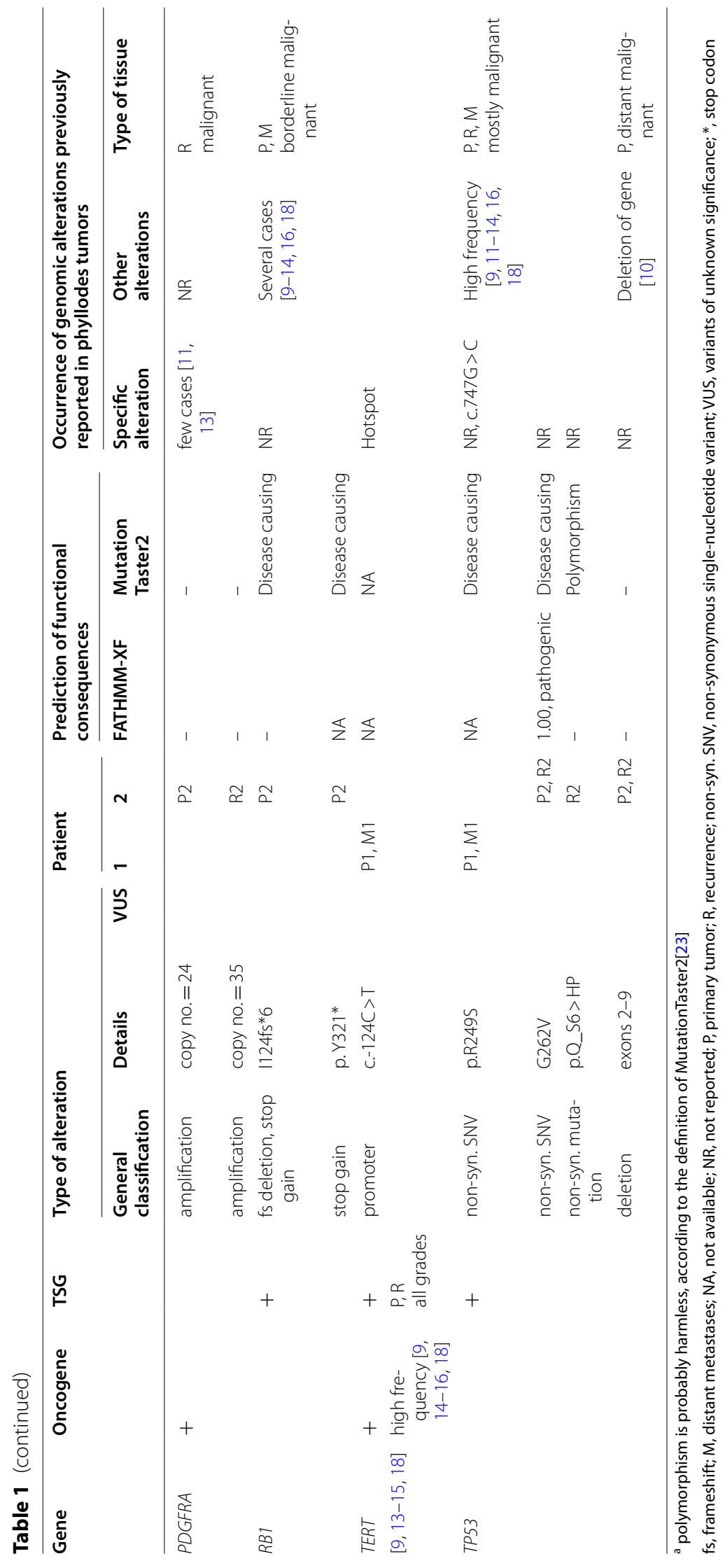


Table 2 Variants of unknown significance identified in the primary tumor (P1) and lung metastasis (M1) of Patient 1 as well as the primary (P2) and recurrent tumor (R2) of Patient 2

\begin{tabular}{|c|c|c|c|c|c|c|c|c|c|}
\hline \multirow[t]{2}{*}{ Gene } & \multicolumn{2}{|l|}{ Type of alteration } & \multicolumn{2}{|l|}{ Patient } & \multicolumn{2}{|c|}{$\begin{array}{l}\text { Prediction of functional } \\
\text { consequences }\end{array}$} & \multicolumn{3}{|c|}{$\begin{array}{l}\text { Occurrence of genomic alterations previously } \\
\text { reported in PTs }\end{array}$} \\
\hline & $\begin{array}{l}\text { General } \\
\text { classification }\end{array}$ & Details & 1 & 2 & FATHMM-XF & MutationTaster2 & $\begin{array}{l}\text { Specific } \\
\text { alteration }\end{array}$ & Other alterations & Type of tissue \\
\hline EPHA3 & non-syn. SNV & p.K713T & $\mathrm{P} 1, \mathrm{M} 1$ & & 0.30 , benign & Disease causing & NR & $N R$ & \\
\hline EPHA7 & non-syn. SNV & p.T118A & M1 & & 0.13 , benign ${ }^{1}$ & Disease causing & NR & NR & \\
\hline$E P H B 1$ & non-syn. SNV & p.R637H & $\mathrm{P} 1, \mathrm{M} 1$ & & 0.06 , benign ${ }^{1}$ & Disease causing & NR & NR & \\
\hline GRM3 & non-syn. SNV & p. N516S & & $P 2, R 2$ & NA & Disease causing & NR & $N R$ & \\
\hline MAF & non-syn. SNV & p.L138M & P1 & & 0.07 , benign & Polymorphism & NR & NR & \\
\hline MSTIR & non-syn. SNV & p.R470H & & $\mathrm{P} 2, \mathrm{R} 2$ & 0.06 , benign ${ }^{1}$ & Disease causing & NR & NR & \\
\hline NTRK3 & non-syn. SNV & p.S564C & $\mathrm{P} 1, \mathrm{M} 1$ & & $\begin{array}{l}\text { 0.02, benign } \\
\text { high confi- } \\
\text { dence }\end{array}$ & Polymorphism & NR & $N R$ & \\
\hline $\begin{array}{l}\text { PRKN } \\
\text { (PARK2) }\end{array}$ & non-syn. SNV & p.R442G & & $P 2, R 2$ & 0.03 , benign & Polymorphism & NR & NR & \\
\hline PIK3C2G & non-syn. SNV & p.H1274D & & $P 2, R 2$ & NA & Disease causing & NR & $\begin{array}{l}\text { One case, non-syn. } \\
\text { SNV [29] }\end{array}$ & $\begin{array}{l}\text { metastatic malig- } \\
\text { nant }\end{array}$ \\
\hline PLCG2 & non-syn. SNV & p.T961M & M1 & & NA & Disease causing & NR & NR & \\
\hline SPTA1 & non-syn. SNV & p.R885H & M1 & & NA & Polymorphism & NR & NR & \\
\hline ZNF703 & $\begin{array}{l}\text { fs deletion, stop } \\
\text { gain mutation }\end{array}$ & p.G22fs*50 & $\mathrm{P} 1, \mathrm{M} 1$ & & - & Disease causing & $N R$ & Three cases [18] & $\begin{array}{l}\text { P, R: benign, } \\
\text { malignant }\end{array}$ \\
\hline
\end{tabular}

fs, frameshift; non-syn. SNV, non-synonymous single-nucleotide variant; NA, not available; NR, not reported; P, primary tumor; PT, phyllodes tumor; R, recurrence; $M$, distant metastases; ${ }^{*}$, stop codon

${ }^{1}$ According to the COSMIC database (cancer.sanger.ac.uk) [22], the FATHMM-MKL prediction was pathogenic for EPHA7 (score: 0.94), EPHB1 (score: 0.99), and MST1R (score: 0.95)

In Patient 2, TMB was 0 mut/Mb in the untreated primary tumor (P2) and 4 mut/Mb in the paired recurrent tumor (R2). Seventeen (P2) and 15 (R2) sequence variants were identified, of which 13 were present in both the primary and progressed tumor sample (Tables 1 and 2, Fig. 3). In both P2 and R2, amplification of the RTKs IGFR1, KIT, and PDGFRA as well as the transcription factor $M Y C$ occurred (Table 1). Moreover, inactivating alterations, such as the deletion of exons 2-9 of TP53 and nonsense mutations in $R B 1$, as well as a SNV in the transcription factor $P A X 5$ were found in both specimens. Interestingly, amplification of EGFR was only identified in the recurrent but not in the primary tumor, suggesting that this might be a crucial event in the evolution of this tumor. Besides alterations in these cancer hallmark genes, SNVs were identified in the less characterized genes GRM3, MST1R, PRKN, and PIK3C2G (Table 2). Mutations in genes confined to P2 (BCOR and RB1) were predicted to be probably disease-causing but only present in low frequencies (Fig. 3, Additional file 1: Table S1).

Four druggable targets (amplification of EGFR, KIT, and PDGFRA; and the missense mutation in EGFR) were identified, leading to proposed off-label therapy with the EGFR antibodies cetuximab and panitumumab, several tyrosine kinase inhibitors such imatinib, nilotinib, sunitinib, and afatinib as well as the multi-kinase inhibitor sorafenib. However, due to R0 resection with wide resection margins in Patient 2 and no sign of progressive disease up until April 2021, these recommendations have not yet been adopted.

To the best of our knowledge (Tables 1 and 2), sequence variants in 15 genes found in the two patients described here (APC, BRIP1, CASP8, CDK4, GRIN2A, EPHA3, EPHA7, EPHB1, GRM3, MAF, MST1R, NTRK3, PRKN, PLCG2, and SPTA1) have not previously been associated with PTs. Five of these were in either oncogenes or TSGs. The judgement as to whether genetic alterations were previously reported in PTs was based on the cited references and 561 phyllodes tumors listed in the COSMIC database as of 04/2021 (cancer.sanger.ac.uk) [22].

\section{Prediction of functional consequences}

In 12 out of 25 instances, analysis of a mutation based on the chromosomal location relative to the Genome Reference Consortium Human (GRCh) 38/hg38, as indicated in the raw data of the NGS report (Additional file 1: Table S1), could not be performed by FATHMM-XF as an unexpected reference base was found and therefore results were not available (Tables 1 and 2). MutationTaster2 could make predictions for 


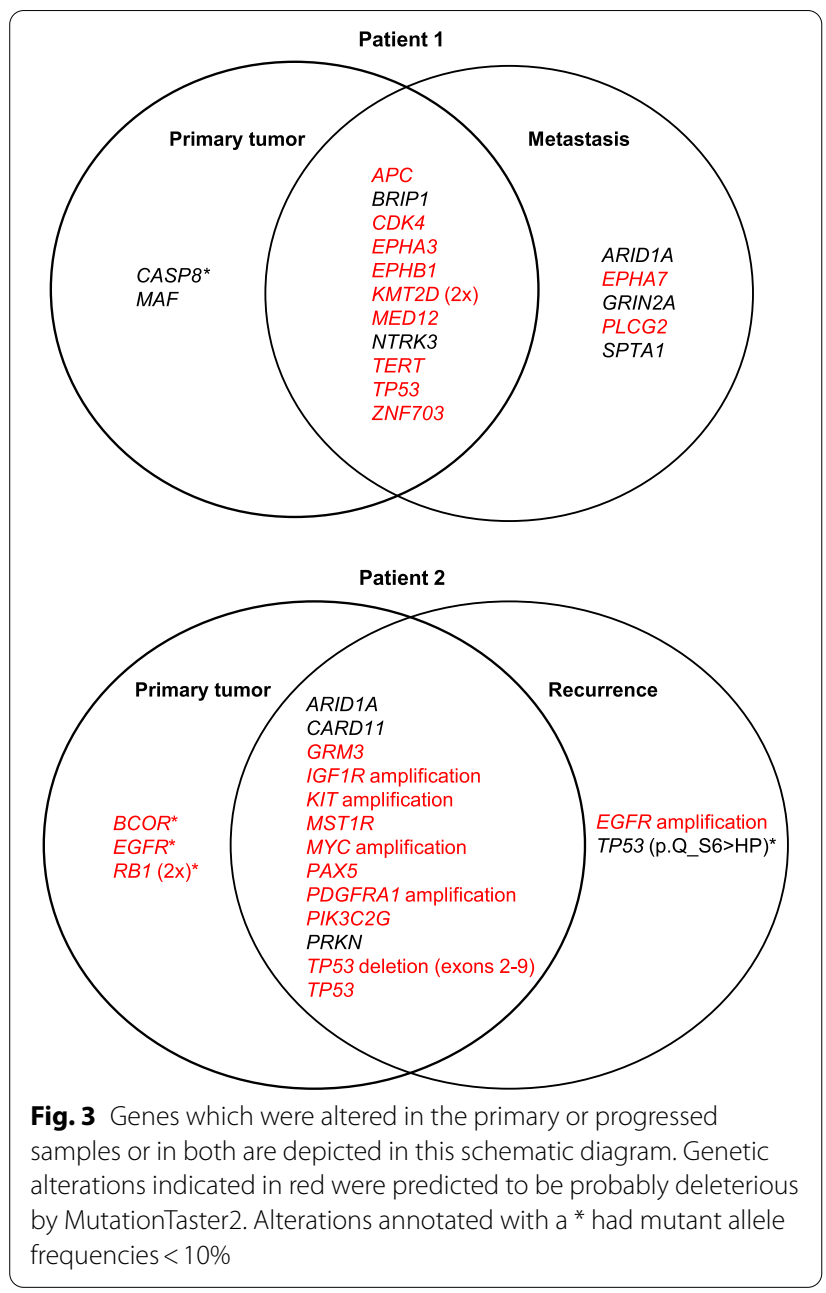

all mutations except the one in the TERT promoter region. Eleven of a total of 38 genetic alterations were found to be probably benign and harmless by MutationTaster2 (e.g. BRIP1, CARD11, CASP8, NTRK3, PRKN, and $S P A T 1$ ), including both non-frameshift alterations in ARID1A. As MutationTaster 2 was considered to predict benignity with high reliability [27], we regarded these predictions as valid. For four of these 11 cases, a FATHMM-XF prediction was available and benign as well. One of the concordant benign predictions with high confidence was for p.M1V in BRIP1 as detected in Patient 1 . The next start codon is at codon 4 , and the delayed start of translation possibly results in a shortened protein containing 1247 instead of 1250 amino acids.

The FATHMM-XF score predicted eight SNVs to be benign, whereas Mutationtaster2 classified four of these eight as probably deleterious. Of note, FATHMM-MKL predictions for three of these discordant predictions were available in the COSMIC database and all were pathogenic: EPHA7 (score: 0.94), EPHB1 (score: 0.99), and MSTR1 (score: 0.95) (Table 2).

\section{Discussion}

The application of modern sequencing technologies for the assessment of genomic alterations and actionable targets is especially valuable for rare cancers such as PT, for which the genetic drivers are poorly understood. The present report describes the results of NGS and prediction of functional consequences of identified genetic alterations for paired samples (P1, M1; P2, R2) of two patients with MPTs.

Except for alterations in ARID1A and TP53, which were present in all samples, the genomic aberrations of the MPTs from Patients 1 and 2 were quite different from each other. The DNA-binding protein ARID1A is involved in the regulation of chromatin architecture [28]. It was predicted that the ARID1A non-frameshift (nonfs) deletion in M1 and the non-fs insertion in P2 and R2 probably lacked functional consequences. Three different genomic alterations in TP53 were found in tumor samples from Patient 2. One of these was a deletion of exons $2-9$, very likely resulting in a non-functional protein or no TP53 protein at all. TP53 is a classic TSG involved in many cancer types [29] including PT [10]. It has been suggested that TP53 alterations play a role in PT progressing from a benign to a malignant histological subtype [12]. TP53 mutations have been detected mainly in malignant and, to a lesser degree, in borderline PTs [9-13].

Among other alterations, P1 and M1 harbored PT hotspot mutations in MED12 [11, 13, 18] and the TERT promoter region $[14,15]$ as well as SNVs in $A P C$ and $C D K 4$. The latter are commonly reported in other advanced cancer entities [29] but, to our knowledge, not yet in PTs. It has been pointed out that uterine adenosarcomas and PTs of the breast are both fibroepithelial lesions harboring mutations in members of the $\mathrm{Wnt} / \beta$-catenin signaling pathway [17]. The same was true for M1, which had genomic alterations in pathway members $A P C, T E R T$, and MED12. MutationTaster2 indicated that the missense mutations detected in EPHA3, EPHA7, and $E P H B 1$ were probably disease-causing. The identical SNV (p.R637H, c.1910G >A) in EPHB1 was previously reported in a patient with breast cancer [30]. Likewise, the identical SNV (p.T118A, c. 352A > G) in EPHA7 was reported in a colorectal cancer cell line [31]. These Eph receptors belong to a family of 14 RTKs [32], and several of these have been associated with cancer and cancer progression [33].

Paired tumor samples from Patient 2 exhibited a very aggressive genomic pattern as four oncogenes (EGFR, $I G F 1 R, M Y C, K I T)$ were amplified and two major TSGs 
(TP53, RB1) were deleted or inactivated, a combination likely to promote genomic instability. In this regard, it is not surprising that a recurrent tumor of $5 \mathrm{~cm}$ evolved within 3 months after the primary diagnosis and 2 months after a CT documented no suspicious findings in the axillary region. In other investigations, amplifications of EGFR [9, 14, 18], IGF1R [11], KIT [13], MYC [10, $11,13,18]$, and PDGFRA $[11,12]$ were confined to MPTs rather than benign or borderline PTs. In fact, it was demonstrated that gene copy alterations were generally associated with higher histological grade [17], suggesting a critical role in the progression of MTPs. Interestingly, four genes encoding RTKs were amplified in Patient 2. A potential actionable target for MPTs is EGFR, which can be blocked by lapatinib. This may offer a potential later therapeutic option for Patient 2, who until now did not show any sign of recurrent disease. In a previous investigation of MPTs, EGFR amplification occurred in 8/24 (33\%) of cases, while nearly all of them exhibited EGFR protein overexpression [12], suggesting that anti-EGFR therapy could become one of the cornerstones for treatment of MPTs with relevant alterations.

Several of the 12 VUS in genes not classified as TGSs or oncogenes (Table 2) were classified as probably harmless by functional annotation scores. Aberrations for ZNF703 and GRM3 were predicted to be probably disease-causing. GRM3 is a G-protein-coupled receptor located upstream of PI3K and the Ras/Raf/MEK/ERK pathway [34]. Of note, none of the 12 VUS reported here has been described in PTs so far, except for PIK3C2G and ZNF703 [18], which belong to the zinc finger protein family of transcription factors. In vitro experiments have shown that ZNF703 is a negative regulator of $\mathrm{Wnt} / \mathrm{B}$-catenin signaling [35], suggesting that this pathway might play an important role in the carcinogenesis of MPTs.

In a huge NGS project including more than 10,000 patients with metastatic cancer across 62 principal solid tumor entities, the most commonly mutated genes were, in decreasing order of alteration frequency, TP53, KRAS, TERT, PIK3CA, APC, ARID1A, PTEN, EGFR, KMT2D, and $R B 1$ [29]. Whereas seven of these genes (TP53, TERT, APC, ARID1A, EGFR, KMT2D, and RB1) were also altered in the two MPTs described here, PTEN, KRAS and PIK3CA had wild-type status. However, PIK3CA was reported to be mutated in a mixed cohort of patients with primary and metastatic MPT cases $[9,12]$, and PIK3C2G (mutated in P2, R2) belongs, like PIK3CA, to the PI3K family. PTEN is an upstream regulator of the PI3K/ AKT/mTOR signaling pathway [36], and was previously shown to be mutated in MPTs [13]. In addition, ligandindependent upregulation of RTKs (e.g. EGFR, PDGFR, $K I T)$ due to gain-of-function mutations (in P2 and R2) can result in constitutive downstream activation of the
Ras/Raf/MEK/ERK pathway, ultimately leading to proliferation and apoptosis resistance [37]. Therefore, despite the limited knowledge regarding underlying mutational events in MPTs, it seems that most of the major genomic alterations frequently occurring in other advanced cancer types are involved in this process as well. These findings should be confirmed in a larger cohort.

A limitation of our evaluation is that the application of in silico prediction tools is not without controversy; a main point of criticism is that predictions might be false positives in some instances even when several prediction tools were applied [27]. In one study, which tested the performance of four prediction tools, MutationTaster2 predicted no false-negative results and was therefore considered a suitable algorithm to predict benignity [27]. Regarding practicability, MutationTaster2 definitely outperformed FATHMM-XF as it predicted functional consequences in 34 of $35 \mathrm{SNVs}$, insertions or deletions, whereas the FATHMM-XF score could only be obtained for 13 out of 25 SNVs. These findings are based on raw data we requested for the original FoundationOne ${ }^{\circledR}$ $(\mathrm{CDx})$ reports; we therefore do not have further insight into the functionality of this prediction tool. Despite the drawbacks associated with these algorithms, especially for clinical decision-making, they added further information to NGS results presented here. Prediction of functional consequences was especially helpful for the interpretation of VUS and particularly in genes which have not been associated with MPTs and/or whose role in carcinogenesis in general is not well described yet.

\section{Conclusions}

Analysis of data generated by NGS provided new insights into the molecular pathogenesis of recurrent and metastatic MPTs, identified novel mutations involved in the progression of MPTs, discovered remarkable similarity with the 10 most frequently altered genes in other advanced cancer entities, and suggested potential therapeutic options. Merely listing genomic alterations without functional analysis could be misleading, as several alterations seem to be benign and might have no role in the pathogenesis of PT.

\section{Supplementary Information}

The online version contains supplementary material available at https://doi. org/10.1186/s13023-021-01986-z.

Additional file 1: Table S1. Details of genetic alterations identified in the primary tumor ( $\mathrm{P} 1)$ and lung metastasis (M1) of Patient 1 and the primary (P2) and recurrent (R2) tumors of Patient 2, based on the Ch38 (hg38) version of the human genome assembly.

Acknowledgements

The authors would like to thank the patients. 


\section{Authors' contributions}

SK, EB, and MR contributed to data collection, interpretation, literature searching, manuscript drafting and critical review of the manuscript. IT contributed pathology images and interpretation of those. DM contributed radiology images and interpretation of those. SB, $\mathrm{HH}, \mathrm{OC}$, and DS contributed to data collection. MB contributed to interpretation of genetic data. All authors read and approved the final manuscript draft.

\section{Funding}

This research received no funding.

\section{Availability of data and materials}

All data generated or analyzed during this study are included in this published article [and its supplementary information files].

\section{Declarations}

\section{Ethics approval and consent to participate}

According to $\$ 15$ of the Nordrhein-Westfalen (Germany) Medical Association professional code of conduct, retrospective studies do not require ethics committee approval. Patients provided written informed consent.

\section{Consent for publication}

The two patients presented here agreed to their anonymous data being published and shared.

\section{Competing interests}

SK reports personal fees from Lilly, Roche, Genomic Health, Novartis, Amgen, Celgene, Daiichi Sankyo, AstraZeneca, Somatex, MSD, Pfizer, Puma Biotechnology, PFM medical, non-financial support from Roche, Daiichi Sankyo, Lilly, and Sonoscape, outside the submitted work. SK is co-director of the WSG Study group. MR reports personal fees and travel support from Novartis and from Pfizer, personal fees from Somatex, MSD, AstraZeneca, Roche, Lilly and Seagen, travel support from Celgene, outside the submitted work. EB reports personal fees from Sysmex, Olympus, Merck Serono, and Daiichi Sankyo, outside the submitted work. All other authors have nothing to disclose.

\section{Author details}

${ }^{1}$ Breast Unit, Kliniken Essen-Mitte, 45136 Essen, Germany. ${ }^{2}$ Department of Gynecology with Breast Center, Charité - Universitätsmedizin Berlin, Corporate Member of Freie Universität Berlin and Humboldt- Universität zu Berlin, 10117 Berlin, Germany. Institute for Pathology Viersen, 41747 Viersen, Germany. ${ }^{4}$ Nuclear Medicine Centre, 45136 Essen, Germany. ${ }^{5}$ Teilgemeinschaftspraxis Molekularpathologie Südbayern, 81539 München, Germany.

\section{Received: 27 April 2021 Accepted: 27 July 2021}

Published online: 16 August 2021

\section{References}

1. Strode M, Khoury T, Mangieri C, Takabe K. Update on the diagnosis and management of malignant phyllodes tumors of the breast. The Breast. 2017;33:91-6.

2. Tan BY, Acs G, Apple SK, Badve S, Bleiweiss IJ, Brogi E, et al. Phyllodes tumours of the breast: a consensus review. Histopathology. 2016;68:5-21.

3. Zhang Y, Kleer CG. Phyllodes tumor of the breast: histopathologic features, differential diagnosis, and molecular/genetic updates. Arch Pathol Lab Med. 2016;140:665-71.

4. Kapiris I, Nasiri N, A'Hern R, Healy V, Gui GP. Outcome and predictive factors of local recurrence and distant metastases following primary surgical treatment of high-grade malignant phyllodes tumours of the breast. Eur J Surg Oncol. 2001;27:723-30.

5. Testori A, Meroni S, Errico V, Travaglini R, Voulaz E, Alloisio M. Huge malignant phyllodes breast tumor: a real entity in a new era of early breast cancer. World J Surg Oncol. 2015;13:81.

6. Koh VCY, Thike AA, Tan PH. Distant metastases in phyllodes tumours of the breast: an overview. Appl Cancer Res. 2017;37:15.
7. Chao X, Chen K, Zeng J, Bi Z, Guo M, Chen Y, et al. Adjuvant radiotherapy and chemotherapy for patients with breast phyllodes tumors: a systematic review and meta-analysis. BMC Cancer. 2019;19:372.

8. Papas Y, El Asmar A, Ghandour F, Hajj I. Malignant phyllodes tumors of the breast: A comprehensive literature review. Breast J. 2020;26:240-4.

9. Liu S-Y, Joseph NM, Ravindranathan A, Stohr BA, Greenland NY, Vohra $P$, et al. Genomic profiling of malignant phyllodes tumors reveals aberrations in FGFR1 and PI-3 kinase/RAS signaling pathways and provides insights into intratumoral heterogeneity. Mod Pathol. 2016;29:1012-27.

10. Jardim DLF, Conley A, Subbiah V. Comprehensive characterization of malignant phyllodes tumor by whole genomic and proteomic analysis: biological implications for targeted therapy opportunities. Orphanet J Rare Dis. 2013:8:112.

11. Cani AK, Hovelson DH, McDaniel AS, Sadis S, Haller MJ, Yadati V, et al. Next-gen sequencing exposes frequent MED12 mutations and actionable therapeutic targets in phyllodes tumors. Mol Cancer Res. 2015;13:613-9.

12. Gatalica Z, Vranic S, Ghazalpour A, Xiu J, Ocal IT, McGill J, et al. Multiplatform molecular profiling identifies potentially targetable biomarkers in malignant phyllodes tumors of the breast. Oncotarget. 2016;7:1707-16.

13. Nozad S, Sheehan CE, Gay LM, Elvin JA, Vergilio J-A, Suh J, et al. Comprehensive genomic profiling of malignant phyllodes tumors of the breast. Breast Cancer Res Treat. 2017;162:597-602.

14. Piscuoglio S, Ng CK, Murray M, Burke KA, Edelweiss M, Geyer FC, et al. Massively parallel sequencing of phyllodes tumours of the breast reveals actionable mutations, and TERT promoter hotspot mutations and TERT gene amplification as likely drivers of progression. J Pathol. 2016;238:508-18.

15. Yoshida M, Ogawa R, Yoshida H, Maeshima A, Kanai Y, Kinoshita T, et al. TERT promoter mutations are frequent and show association with MED12 mutations in phyllodes tumors of the breast. Br J Cancer. 2015;113:1244-8

16. Pareja F, Geyer FC, Kumar R, Selenica P, Piscuoglio S, Ng CKY, et al. Phyllodes tumors with and without fibroadenoma-like areas display distinct genomic features and may evolve through distinct pathways. NPJ Breast Cancer. 2017;3:40.

17. Geyer FC, Burke KA, Piscuoglio S, Ng CKY, Papanastasiou AD, Marchiò C, et al. Genetic analysis of uterine adenosarcomas and phyllodes tumors of the breast. Mol Oncol Wiley Blackwell. 2017;11:913-26.

18. Kim J-Y, Yu JH, Nam SJ, Kim SW, Lee SK, Park W-Y, et al. Genetic and clinical characteristics of phyllodes tumors of the breast. Transl Oncol. 2018;11:18-23.

19. Garcia-Dios DA, Levi D, Shah V, Gillett C, Simpson MA, Hanby A, et al. MED12, TERT promoter and RBM15 mutations in primary and recurrent phyllodes tumours. Br J Cancer Nature Publishing Group. 2018;118:277-84.

20. Takeuchi S, Okuda S. Knowledge base toward understanding actionable alterations and realizing precision oncology. Int J Clin Oncol. 2019;24:123-30.

21. van der Velden $\mathrm{DL}$, van Herpen $C M L$, van Laarhoven HWM, Smit EF, Groen HJM, Willems SM, et al. Molecular Tumor Boards: current practice and future needs. Ann Oncol. 2017;28:3070-5.

22. Tate JG, Bamford S, Jubb HC, Sondka Z, Beare DM, Bindal N, et al. COSMIC: the catalogue of somatic mutations in cancer. Nucleic Acids Res Oxford University Press. 2019;47:D941-7.

23. Schwarz JM, Cooper DN, Schuelke M, Seelow D. MutationTaster2: mutation prediction for the deep-sequencing age. Nat Methods. 2014;11:361-2.

24. Rehm HL, Berg JS, Plon SE. ClinGen and ClinVar-enabling genomics in precision medicine. Hum Mutat. John Wiley and Sons Inc: 2018;39:1473-5.

25. Rogers MF, Shihab HA, Mort M, Cooper DN, Gaunt TR, Campbell C. FATHMM-XF: accurate prediction of pathogenic point mutations via extended features. Hancock J, editor. Bioinformatics. Narnia; 2018;34:511-3.

26. Frampton GM, Fichtenholtz A, Otto GA, Wang K, Downing SR, He J, et al. Development and validation of a clinical cancer genomic profiling test based on massively parallel DNA sequencing. Nat Biotechnol. 2013;31:1023-31

27. Ernst C, Hahnen E, Engel C, Nothnagel M, Weber J, Schmutzler RK, et al. Performance of in silico prediction tools for the classification of rare 
BRCA1/2 missense variants in clinical diagnostics. BMC Med Genomics. 2018;11:35.

28. Wu JN, Roberts CWM. ARID1A mutations in cancer: another epigenetic tumor suppressor? . Cancer Discov. 2013;3:35-43.

29. Zehir A, Benayed R, Shah RH, Syed A, Middha S, Kim HR, et al. Mutational landscape of metastatic cancer revealed from prospective clinical sequencing of 10,000 patients. Nat Med. 2017;23:703-13.

30. Ellis MJ, Ding L, Shen D, Luo J, Suman VJ, Wallis JW, et al. Whole-genome analysis informs breast cancer response to aromatase inhibition. Nature. 2012;486:353-60

31. Mouradov D, Sloggett C, Jorissen RN, Love CG, Li S, Burgess AW, et al. Colorectal Cancer Cell Lines Are Representative Models of the Main Molecular Subtypes of Primary Cancer. Cancer Res. 2014;74:3238-47.

32. Liang L-Y, Patel O, Janes PW, Murphy JM, Lucet IS. Eph receptor signalling: from catalytic to non-catalytic functions. Oncogene. 2019;38:6567-84.

33. Darling TK, Lamb TJ. Emerging roles for eph receptors and ephrin ligands in immunity. Front Immunol. 2019;10.
34. Prickett TD, Samuels Y. Molecular pathways: dysregulated glutamatergic signaling pathways in cancer. Clin Cancer Res. 2012;18:4240-6.

35. Kumar A, Chalamalasetty RB, Kennedy MW, Thomas S, Inala SN, Garriock $\mathrm{RJ}$, et al. Zfp703 Is a Wnt/B-catenin feedback suppressor targeting the ß-catenin/Tcf1 complex. Mol Cell Biol. 2016;36:1793-802.

36. Papa P. The PTEN-PI3K axis in cancer. Biomolecules. 2019;9:153.

37. Montor WR, Salas AROSE, de Melo FHM. Receptor tyrosine kinases and downstream pathways as druggable targets for cancer treatment: the current arsenal of inhibitors. Mol Cancer. 2018;17:55.

38. Tan J, Ong CK, Lim WK, Ng CCY, Thike AA, Ng LM, et al. Genomic landscapes of breast fibroepithelial tumors. Nat Genet. 2015;47:1341-5.

\section{Publisher's Note}

Springer Nature remains neutral with regard to jurisdictional claims in published maps and institutional affiliations.
Ready to submit your research? Choose BMC and benefit from:

- fast, convenient online submission

- thorough peer review by experienced researchers in your field

- rapid publication on acceptance

- support for research data, including large and complex data types

- gold Open Access which fosters wider collaboration and increased citations

- maximum visibility for your research: over 100M website views per year

At BMC, research is always in progress.

Learn more biomedcentral.com/submissions 